\title{
Carbon Nanotube Nanoradios : The Field Emission and Transistor Configurations
}

\author{
P. Vincent, ${ }^{*}$ A. Ayari, P. Poncharal, T. Barois, S. Perisanu, V. Gouttenoire, and S.T. Purcell \\ Laboratoire de Physique de la Matière Condensée et Nanostructures, Université Lyon 1, \\ CNRS, UMR 5586, Domaine Scientifique de la Doua, F-69622 Villeurbanne Cedex, France.
}

\begin{abstract}
In this paper, we explore and compare two distinct configurations of the "nanoradio" concept where individual carbon nanotube resonators are the central electro-mechanical element permitting signal demodulation. The two configurations of singly-clamped field emitters and doubly-clamped field effect transistors are examined which at first glance are quite different, but in fact involve quite similar physical concepts. Amplitude, frequency and digital demodulation are demonstrated and the analytical formulae describing the demodulation are derived as functions of the system parameters. The crucial role played by the mechanical resonance in demodulation is clearly demonstrated. For the field emission configuration we particularly concentrate on how the demodulation depends on the variation of the field amplification factor during resonance and show that amplitude demodulation results in the best transmitted signal. For the transistor configuration the important aspect is the variation of the nanotube conductance as a function of it's distance to the gate. In this case frequency demodulation is much more effective and digital signal processing was achieved. The respective strengths and weaknesses of each configuration are discussed throughout the article.
\end{abstract}

PACS numbers: $85.85 .+\mathrm{j}, 61.48 . \mathrm{De}, 79.70 .+\mathrm{q}, 84.30 . \mathrm{Qi}$

\section{Introduction}

Electromechanical resonators are a key element in radio-frequency telecommunications devices and thus new resonator concepts from nanotechnology can readily find important industrial opportunities. Carbon nanotubes (CNTs) have nanometric lateral dimensions and well-defined mechanical resonance frequencies in the $\mathrm{MHz}$ to $\mathrm{GHz}$ range. These resonances can be excited and detected through electrical signals, thus making CNTs ideal candidates for discovering and exploring new concepts in RF nanotechnology. They may lead to extremely compact RF components that can potentially open applications in smart dust components ${ }^{1}$, enhanced radio frequency identification (RFID) tags or simply smaller and cheaper wireless devices such as cellular phones.

The time-line of critical references for this work starts with the first experiments on the electrical excitation and observation of the mechanical resonances of multi-wall CNTs (MWNTs) ${ }^{2}$. The MWNTs were attached at one end, i.e. single-clamped, and the experiments were carried out in a transmission electron microscope (TEM). The excitation was done by applying RF signals to the tip support or a counter electrode and the oscillations were observed by TEM imaging. The electrical excitation of the resonances of MWNTs undergoing field emission (FE) were then observed by the variation in the FE current and patterns ${ }^{3}$. The large axial force generated by the CNT apex electric field was found to tune the mechanical resonances over a wide range. The resonances of suspended single-wall nanotube (SWNTs), i.e. doubleclamped, acting as the channel of field effect transistors were studied by down-mixing techniques. ${ }^{4}$ The resonance frequencies could again be electrically tuned, in this case with transverse forces generated by the gate voltage, as originally proposed ${ }^{3,5}$. Jensen, et al. ${ }^{6}$ used the singleclamped TEM configuration $^{2}$, the FE technique ${ }^{3,7}$ and the notions of down-mixing ${ }^{4}$ to demonstrate the "nanora- dio" concept where MWNT resonators under FE could process radio signals, even decoding music. Similar music decoding experiments were carried out on non-suspended SWNT transistors ${ }^{8}$ but which could not have a mechanical resonance to select a particular carrier frequency. The nanoradio was extended to suspended CNT field effect transistors for which amplitude, frequency or digitally demodulation (AM, FM, DM) using the mechanical resonance ${ }^{9}$ were investigated. Recently a detailed, quantitative theoretical and experimental study of FE nanoradio has been submitted ${ }^{10}$.

The singly-clamped FE resonator has led to several original research subjects besides the nanoradio including position sensors ${ }^{11}$, atomic mass sensing ${ }^{12}$, self-oscillating AC nanogenerators ${ }^{13}$ and the FE electron shuttle ${ }^{14}$ while the double clamped geometry has been recently used for studying phase transitions of surface adsorbates ${ }^{15}$ and single electron charging of SWNT resonators ${ }^{16,17}$.

In this article we review our recent work ${ }^{9,10}$ on RF signal processing with resonating CNTs in the two configurations. The singly-clamped FE configuration is shown in figure 1(a). A CNT is attached mechanically and electrically on one end to a support structure. The application of a large applied voltage $\left(V_{A}\right)$ and the tip shape induces a large field $F=\beta V_{A}$ at the cap end where $\beta$ is the geometrical field amplification factor. This induces a FE tunnel current, $I_{F E} \alpha \exp (-$ const $/ F)$, from the cap. An incoming electrical signal $(S(t))$, which contains a harmonic component near the mechanical resonance frequency, can induce vibrations by the surface forces they generate on the $\mathrm{CNT}^{2}$. This in turn causes simultaneous oscillations in $\beta \rightarrow \beta(t)$ and $I_{F E} \rightarrow I_{F E}(t)$, thus transducing $S(t)$ only near the resonance frequency. $S(t)$ can be AM, FM and DM modulated and thus information can be transmitted. The key parameter is the dependence of $I_{F E}$ on the position of the open end, $y(t)$, through the field amplification factor, i.e. $I_{F E}(\beta(y(t)))$. Note that 


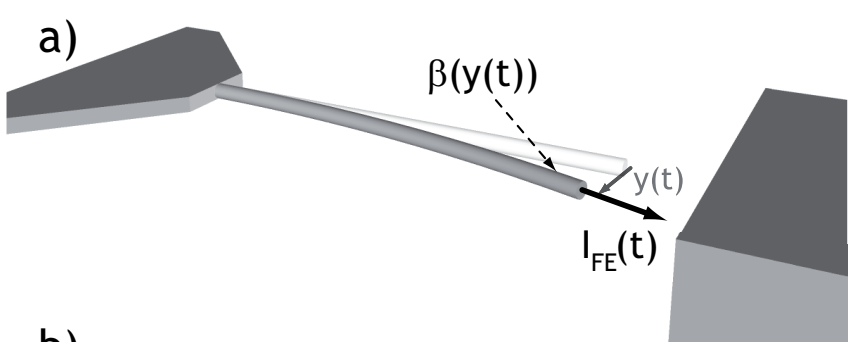

b)



FIG. 1: (Color online) Schematic of the two nanoradio configurations. (a) the single-clamped FE nanoradio. (b) the double-clamped suspended SWNT transistor nanotube.

we concentrate on carbon nanotubes for now rather than nanowires because they presently allow much higher and stabler field emission currents which are necessary for this application. This is intimately related to their material and geometrical properties: the high chemical and thermal stability of carbon itself, the relatively high electrical and thermal conductivities when using high quality nanotubes, the readily available and now easily handled CNTs with low diameters and high aspect ratios which allow lower voltage field emission and particularly the electrical tuning of the mechanical resonances.

The second configuration is the CNT field effect transistor (figure 1(b)) in which a single wall nanotube (SWNT) is suspended between the source and drain electrodes and over a planar gate. The gate voltage $\left(V_{g}\right)$ controls the source drain current $I_{s d}$ and also provides a mechanical lateral force on the nanotube. An incoming electrical signal again induces vibrations near the mechanical resonance frequency. In this case the key parameter is the variation in conductivity as a function of the lateral position $y(t)$ of the nanotube with respect to the gate, which induces variations in $I_{s d}(y(t))$. SWNTs are used as opposed to MWNTs because only they can be semiconductors which is necessary for the transistor effect.

The article is organized as follows. The next section contains the theoretical basis. We first review the response of a mechanical oscillator to AM and FM excitation and the accompanying demodulation in a general way. Then small signal theory is used to write explicit equations for the demodulated parts of $I_{F E}$ and $I_{s d}$.
The analysis for the single-clamped configuration goes into more detail because $\beta(y)$ can presently be better quantified than the equivalent parameters in the doubleclamped configuration. The experimental results are collected in the next section followed by a discussion and concluding remarks.

\section{Theory}

\section{Mechanical demodulation}

The equations that govern the two configurations are both based on radio science principles. We concentrate on linear small signal analysis of demodulation. The mechanical excitation of CNTs by pure sinusoidal high frequency signals has been investigated in $\mathrm{TEM}^{2}$, scanning electron microscopy ${ }^{18}$ (SEM) and FE environments ${ }^{3,19,20}$ to obtain the angular resonance frequency $\omega_{0}$ (denoted as frequency in the following), the Young's modulus $\mathrm{Y}$ and the quality factors $\mathrm{Q}$ of different CNTs and NWs. For an excitation of the form $V(t)=A \cos (\omega t)$ and a linear mechanical response one has the well-known Lorentzian oscillation amplitude given by (also shown in blue in Fig. 2) :

$$
\begin{gathered}
y(t)=\frac{T}{\sqrt{\left(\omega_{0}^{2}-\omega^{2}\right)^{2}+\left(\omega_{0} \omega / Q\right)^{2}}} \cos (\omega t+\phi) \\
=y_{R} \cos (\omega t)+y_{I} \sin (\omega t)
\end{gathered}
$$

$T$ is the transverse excitation force amplitude per effective unit mass $\left(\mathrm{m} . \mathrm{s}^{-2}\right)$ due to the imbalance of electric fields over the whole CNT surface. It is zero for a perfectly symmetric configuration. For the single-clamped configuration this symmetry is broken if the CNT, the support tip or the counter electrode have non-zero tilt angles for the rest position, or if the CNT is structurally asymmetric. The double-clamped transistor configuration is obviously asymmetric. $y_{R}$ and $y_{I}$ explicitly are the in-phase (or real) and out of phase (or imaginary) response functions given respectively by:

$$
\begin{aligned}
y_{R} & =\frac{\omega_{0}^{2}-\omega^{2}}{\left(\omega_{0}^{2}-\omega^{2}\right)^{2}+\left(\omega_{0 \omega} / Q\right)^{2}} \\
y_{I} & =\frac{\omega_{0} \omega / Q}{\left(\omega_{0}^{2}-\omega^{2}\right)^{2}+\left(\omega_{0} \omega / Q\right)^{2}}
\end{aligned}
$$

Now consider the excitation of the resonance by modulated signals. A pure cosine low frequency modulation is used in the analysis (a pure note). For AM modulation the RF signal is given by

$$
V_{A M}(t)=A\left(1+h \cos \left(\omega_{L} t\right)\right) \cos \left(\omega_{c} t\right)
$$

where $\mathrm{A}$ is the amplitude, $\omega_{L}$ is the frequency of the modulating signal, $\omega_{c}$ is the carrier frequency and $h$ is called the modulation index $(h<1)$ which varies the strength of the modulation.

In FM the modulation is introduced via the phase $\phi(t)$ of the RF signal $V_{F M}(t)=A \cos (\phi(t))$ by the relation 


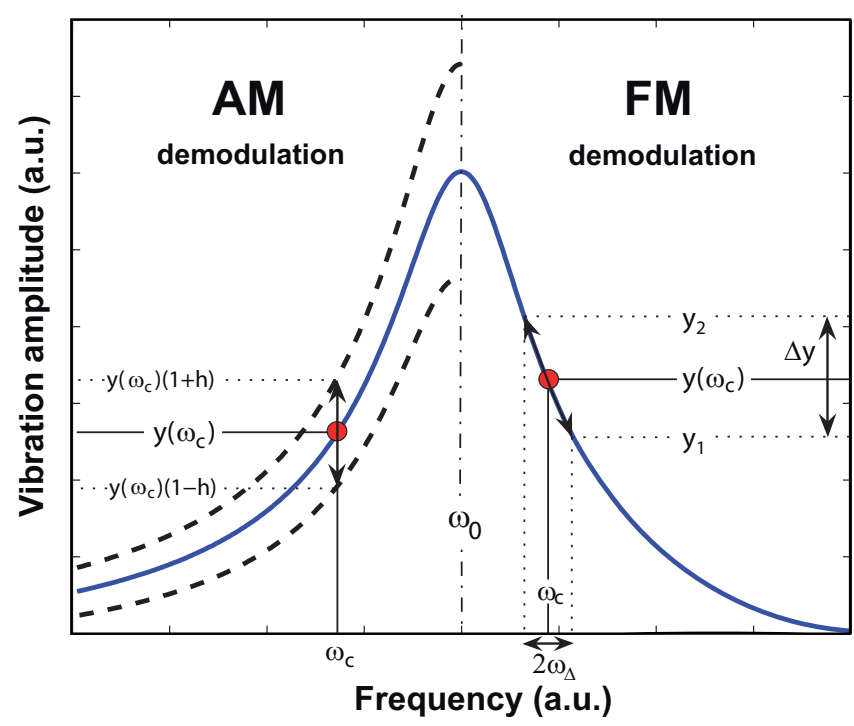

FIG. 2: (Color online) Schematic showing the demodulation process across the mechanical resonance of a CNT. The solid line (blue) traces the linear mechanical amplitude versus the excitation frequency. Left (right) schematizes the AM (FM) demodulation process. The AM and FM signals result in low frequency variations of the oscillations that cause in turn variations of $\beta(y)$.

$\mathrm{d} \phi / d t=\omega_{i}=\omega_{c}+\omega_{\Delta} \cos \left(\omega_{L} t\right)$ where $\omega_{i}$ is the instantaneous frequency, $\omega_{c}$ is again the carrier frequency and $\omega_{\Delta}$ is the frequency deviation that corresponds to the maximum deviation of the instantaneous frequency with respect to the carrier frequency. Integrating $\mathrm{d} \phi / \mathrm{dt}$ one obtains for the FM signal,

$$
V_{F M}(t)=A \cos \left(\omega_{c} t+\omega_{\Delta} / \omega_{L} \sin \left(\omega_{L} t\right)\right)
$$

In a standard $\mathrm{AM}(\mathrm{FM})$ radio, $\mathrm{h}\left(\omega_{\Delta}\right)$ is proportional to the amplitude of the sound and $\omega_{L}$ is its frequency.

It is useful to rewrite equation 6 using the Jacobi-Anger expansion:

$$
\begin{aligned}
V_{F M}(t)= & A\left[J_{0}\left(\frac{\omega_{\Delta}}{\omega_{L}}\right) \cos \left(\omega_{c} t\right)+\sum_{n=1}^{\infty} J_{n}\left(\frac{\omega_{\Delta}}{\omega_{L}}\right)\right. \\
& \left.\left(\cos \left(\left(\omega_{c}-n \omega_{L}\right) t\right)+(-1)^{n} \cos \left(\left(\omega_{c}+n \omega_{L}\right) t\right)\right)\right]
\end{aligned}
$$

where $J_{n}$ is the n-th Bessel function.

It is well known in electronics that a non-linear device, such as a diode or an envelope detector, is sufficient to recover the low frequency part of an AM signal. For example, in principle the strongly non-linear FE characteristic is sufficient for demodulation in configuration 1, independently of the mechanical vibration. Also a nonlinear response of a non-vibrating CNT transistor was used to demodulate ${ }^{8}$. For FM demodulation a specific component must be used whose output varies linearly with the instantaneous frequency of the incoming signal.
The simplest form of FM detector is a slope detector such as a tank circuit which first converts the FM to AM. This AM signal is then demodulated as before. It can be shown that the square of equation 7 , needed for second order terms in Taylor expansions, has no signal at the modulation frequency $\omega_{L}$ and only DC and high frequency signals. Thus pure electrical nonlinearity, as used in ref. ${ }^{8}$, cannot demodulate an FM signal, i.e. no sound can be heard from this term in the FM mode.

An essential element of this work is that the CNT mechanical resonance allows to demodulate at a particular carrier frequency (or radio station) which the simple nonlinear FE and transistor response currents do not allow. The vibrating CNTs provide a supplementary stage, the mechanical resonator, inserted between the electrical excitation and the final demodulated current. This leads to original terms in the mathematical description of both $\mathrm{AM}$ and FM demodulation that strongly increase the resulting demodulated currents.

The effect of the RF modulated signal on the oscillation when the carrier frequency matches the resonance is illustrated in figure 2 for both AM (left of the figure) and FM (right). For AM, the carrier frequency being constant, the oscillation amplitude varies at $\omega_{L}$. For FM the excitation frequency slowly oscillates around the carrier frequency and the amplitude of oscillation follows $y(\omega)$, resulting in an amplitude modulated oscillation. This is similar to an electronic slope detector though the modulation is now mechanical and not electrical.

We now apply standard small signal analysis for $y(\omega)$. For a given carrier frequency and modulation index, the time dependant amplitude of oscillation for an AM signal is given by :

$$
y(t)=y\left(\omega_{c}\right)\left(1+h \cos \left(\omega_{L} t\right)\right) \cos \left(\omega_{c} t+\phi\left(\omega_{c}\right)\right)
$$

where $\phi\left(\omega_{c}\right)$ is the phase shift between the mechanical oscillation and the excitation signal.

For FM let's consider small frequency deviations as shown in figure 2 somewhat off the resonance maximum. It is more convenient to rewrite the excitation signal as $V_{F M}(t)=A \cos \left(\omega_{i} t\right)$ and to consider that the CNT follows the instantaneous frequency, $\omega_{i}$. This approximation is correct here since the transients disappear very rapidly compared to the low frequency modulation. We can then write the evolution of amplitude as (see fig. 2):

$$
y(t)=\left(y\left(\omega_{c}\right)+y^{\prime}\left(\omega_{c}\right) \omega_{\Delta} \cos \left(\omega_{L} t\right)\right) \cos \left(\omega_{i} t+\phi\left(\omega_{i}\right)\right)
$$

where $y^{\prime}\left(\omega_{c}\right)$ is the first derivative of the amplitude with respect to frequency. Thus the FM translates into AM with $h$ replaced by $y^{\prime}\left(\omega_{c}\right) \omega_{\Delta} / y\left(\omega_{c}\right)$.

Now consider the measured signal which in both cases is a current that transits the cantilever. It depends on the transverse position of the cantilever $y(t)$, the amplitude of the RF signal and a static voltage that controls the resonant frequency. We can write in general $I(y(t), R F$ signal, $\omega(V($ control $))$. We will use small signal theory to write explicit equations for $I_{F E}$ and $I_{s d}$ 
in the two cases, particularly the low frequency demodulated component. The single-clamped configuration turns out to require extra analysis because we need to understand $\beta(y)$.

\section{Single-clamped}

As stated above the transverse displacement of the apex of an oscillating $\mathrm{CNT}, \mathrm{y}(\mathrm{t})$, varies $\beta(y)$ and hence $I_{F E}(t)$. The non-linear FE current creates harmonic signals, including the low frequency modulation signal, whose relative importance depends on the variation of $\beta(y)$ around the CNT rest position. In the following we will analyze the frequency response in detail and discuss the different contributions that can be exploited for improving the demodulation.

The next step is to consider how the mechanical oscillations modify $\beta(y)$. Though detailed simulations are presented below we first consider the Taylor expansion of $\beta(y)$ around the CNT apex rest position :

$$
\beta(y)=\beta_{0}+\beta_{1} y+\beta_{2} y^{2}+o\left(y^{2}\right)
$$

$\beta_{1}$ and $2 \beta_{2}$ are the first and second spatial derivatives. They depend strongly on the local environment of the CNT and give different contributions to the demodulated current. In particular it is straightforward that in a symmetric configuration all the odd terms in the development vanish and thus the first term to consider is the second order term. This term is missing in the earlier analysis ${ }^{6}$. The second order term is in fact particularly interesting since its non-linear dependance has a strong demodulation effect.

Substituting eqs. 8 or 9 into 10 , one sees that the $\beta_{1} y$ term gives rise to a high frequency modulated variation although the $\beta_{2} y^{2}$ term contains a demodulated component. In the case of eq. 9 (FM) one gets :

$$
\begin{aligned}
\beta_{2} y^{2} & =\beta_{2}\left(y\left(\omega_{c}\right)+y^{\prime}\left(\omega_{c}\right) \omega_{\Delta} \cos \left(\omega_{L} t\right)\right)^{2} \cos ^{2}\left(\omega_{i} t+\phi\left(\omega_{i}\right)\right) \\
& =c s t+\beta_{2} y\left(\omega_{c}\right) y^{\prime}\left(\omega_{c}\right) \omega_{\Delta} \cos \left(\omega_{L} t\right) \\
& +\frac{\beta_{2}}{4} y^{\prime 2}\left(\omega_{c}\right) \omega_{\Delta}^{2} \cos \left(2 \omega_{L} t\right)+H F \text { terms }
\end{aligned}
$$

This gives respectively a constant term, a low frequency signal at $\omega_{L}$ (the demodulated signal), a low frequency signal at $2 \omega_{L}$ that is the harmonic distortion altering the quality of the signal and high frequency components around $2 \omega_{i}$. The main point here is the appearance of a second order demodulated component in the variation of $\beta$ that in turn will lead to a direct demodulation current.

The final step is to re-inject these variations into the Fowler-Nordheim equation. Here we take a simple but sufficient approximation of the current under the form:

$$
I_{F E}(V, y)=C \beta(y)^{2} V^{2} \exp \left(\frac{-B}{\beta(y) V}\right)
$$

$V=V_{A}+d V$ is the total voltage, $d V$ is the $\mathrm{V}_{A M}$ or $\mathrm{V}_{F M}$ signal and $d \beta$ is the variation in the field amplification factor given by $d \beta=\beta(y)-\beta_{0} . I_{F E}$ can be developed by a Taylor expansion around $\left(V_{A}, \beta_{0}\right)$ as:

$$
\begin{aligned}
I_{F E}(t)= & I_{0}+\frac{\partial I}{\partial V} d V+\frac{\partial I}{\partial \beta} d \beta+\frac{\partial^{2} I}{\partial V^{2}} \frac{d V^{2}}{2} \\
& +\frac{\partial^{2} I}{\partial^{2} \beta} \frac{d \beta^{2}}{2}+\frac{\partial^{2} I}{\partial \beta \partial V} d \beta d V
\end{aligned}
$$

The different partial derivatives can be expressed in terms of FE parameters ${ }^{22}$. The different terms that will contribute to demodulation are contained in this equation.

The first interesting term for AM is the $d \beta$ one since its $\beta_{2} y^{2}$ factor contains a demodulated component. The $d V^{2}$ also contributes non-linearly, the $d \beta^{2}$ demodulates partly due to the $\beta_{1} y$ dependance and finally the $d \beta d V$ also contributes. These additional mechanical resonance terms greatly improve the demodulation performances.

The $d \beta$ term is still present for FM for the same reason as before but the $d V^{2}$ term does not contribute this time. The $d \beta^{2}$ and $d \beta d V$ terms also demodulate.

Eqs 8, 9 and 10 can be used to directly estimate for each term of equation 13 the amplitude of the demodulated current. After removing the $\cos \left(\omega_{L} t\right)$ factors this gives the following AM components :

$$
\begin{aligned}
d \beta: & I_{0}\left(\frac{2}{\beta_{0}}+\frac{B}{\beta_{0}^{2} V_{A}}\right) h \beta_{2} y\left(w_{c}\right)^{2} \\
d \beta^{2}: & I_{0}\left(\frac{2}{\beta_{0}^{2}}+\frac{2 B}{\beta_{0}^{3} V_{A}}+\frac{B^{2}}{\beta_{0}^{4} V_{A}^{2}}\right)\left[h \left(\beta_{1}^{2} y\left(\omega_{c}\right)^{2}\right.\right. \\
& \left.\left.+3 \beta_{2}^{2} y\left(\omega_{c}\right)^{4}\right)+h^{3} \frac{9}{8} \beta_{2}^{2} y\left(\omega_{c}\right)^{4}\right] \\
d V^{2}: & I_{0}\left(\frac{2}{V_{A}^{2}}+\frac{2 B}{\beta_{0} V_{A}^{3}}+\frac{B^{2}}{\beta_{0}^{2} V_{A}^{4}}\right) \frac{A^{2}}{2} \\
d \beta d V: & I_{0}\left(\frac{4}{\beta_{0} V_{A}}+\frac{3 B}{\beta_{0}^{2} V_{A}^{2}}+\frac{B^{2}}{\beta_{0}^{3} V_{A}^{3}}\right) \beta_{1} \times \\
& y_{R}\left(\omega_{c}\right) h \frac{A}{2}
\end{aligned}
$$

$y_{R}\left(\omega_{c}\right)$ is the in-phase response function. This function appears here because in the $d \beta d V$ term the response function is multiplied by the excitation signal which involves the real part of the transfer function.

For FM demodulation the same kind of analysis gives: 


$$
\begin{aligned}
d \beta: & I_{0}\left(\frac{2}{\beta_{0}}+\frac{B}{\beta_{0}^{2} V_{A}}\right) \beta_{2} y\left(w_{c}\right) y^{\prime}\left(w_{c}\right) \omega_{\Delta} \\
d \beta^{2}: & I_{0}\left(\frac{2}{\beta_{0}^{2}}+\frac{2 B}{\beta_{0}^{3} V_{A}}+\frac{B^{2}}{\beta_{0}^{4} V_{A}^{2}}\right) y\left(\omega_{c}\right) \omega_{\Delta}\left[\beta_{1}^{2} y^{\prime}\left(\omega_{c}\right)\right. \\
& \left.+\frac{3}{2} \beta_{2}^{2} y\left(\omega_{c}\right)^{2} y^{\prime}\left(\omega_{c}\right)+\frac{9}{8} \beta_{2}^{2} y^{\prime}\left(\omega_{c}\right)^{3} \omega_{\Delta}^{2}\right] \\
d \beta d V: & I_{0}\left(\frac{4}{\beta_{0} V_{A}}+\frac{3 B}{\beta_{0}^{2} V_{A}^{2}}+\frac{B^{2}}{\beta_{0}^{3} V_{A}^{3}}\right) \beta_{1} \times \\
& y_{R}^{\prime}\left(\omega_{c}\right) \omega_{\Delta} \frac{A}{2}
\end{aligned}
$$

$y_{R}^{\prime}\left(\omega_{c}\right)$ is the first derivative of the in-phase response function.

\section{Double-clamped}

The source current $I_{s d}$ depends on three parameters: the source drain voltage $V_{s d}$, the gate voltage $V_{g}$ and $y$, the position of the middle of the nanotube perpendicular to the substrate. In our experiment, the DC gate voltage was fixed and a pure AC source drain voltage $d V(t)$ was used. The nanotube equilibrium position, defining $y=0$, varies with $V_{g}$ due to the electrostatic bending toward the gate. The vibrations are again described by $y(t)$. The leading terms of the current are given by a second order Taylor expansion around $\left(0,0, V_{g}\right)$ :

$$
\begin{aligned}
I_{s d}\left(V_{s d}(t), y(t), V_{g}\right) & =I_{s d}\left(0,0, V_{g}\right)+\frac{\partial I_{s d}}{\partial V_{s d}} d V+\frac{\partial I_{s d}}{\partial y} y \\
& +\frac{\partial^{2} I_{s d}}{\partial V_{s d}^{2}} \frac{d V^{2}}{2}+\frac{\partial^{2} I_{s d}}{\partial y^{2}} \frac{y^{2}}{2} \\
& +\frac{\partial^{2} I_{s d}}{\partial V_{s d} \partial y} d V y
\end{aligned}
$$

The first, third and fifth terms on the right hand side are zero because no current flows in the absence of source drain voltage, for any $y$ or $V_{g}$. The second term gives only high frequency signals. The higher order terms turn out to be responsible for the low frequency signals that can be detected by the lock-in. The fourth term is the same as the one of ref. ${ }^{8}$. For an applied AM signal, this term gives a DC rectified signal, several high frequency signals and a signal at the modulation frequency. With FM it was pointed out above that for the $d V^{2}$ pure electrical non linearities cannot demodulate an FM signal and thus all the low frequency signal will be related to the vibration. As we will see this turns out to permit better signal to noise ratio in configuration 2 .

The remaining sixth term is proportional to the electromechanical displacement. For AM it has a frequency response similar to the forth term however an important point is that it is the only term that demodulates an FM signal.

It is usually assumed that $\partial^{2} I_{s d} / \partial V_{s d} \partial y$ is proportional to the transconductance and to $C^{\prime}$, the space derivative of the capacitance between the tube and the gate, but it could also be related to a piezoresistive effect. Such piezoresistive effects are possible either due to a strain-induced intrinsic conductance change ${ }^{23,24}$ or a variation of the contact resistance. For generality we do not choose between these models. This makes the analysis seem simpler than the single-clamped geometry but in fact it means that we are not presently able to parameterize $\partial^{2} I_{s d} / \partial V_{s d} \partial y$ and $C^{\prime}$ and thus less insight is available.

The remaining factor to treat is $y$. The driving force for the mechanical vibrations is $C^{\prime}\left(V_{g}-d V\right)^{2} / 2$. After linearization the amplitude of the mechanical response is $T=C^{\prime} V_{g} V_{A} / 2 m_{\text {eff }}$ where $m_{\text {eff }}$ is the effective mass of the CNT. Equations 3, 5 and 6 are used to evaluate the fourth and sixth terms of 21 .

For AM this gives:

$$
\begin{aligned}
d V^{2} & : \frac{1}{2} A^{2} h \frac{\partial^{2} I}{\partial V_{s d}^{2}} \\
y d V & : \frac{A T}{2} \frac{\partial^{2} I}{\partial y \partial V_{s d}} y_{R}\left(\omega_{c}\right) h
\end{aligned}
$$

For FM we have:

$$
y d V: \frac{\partial^{2} I_{s d}}{\partial V_{s d} \partial y} d V y=\frac{A T}{2} \frac{\partial^{2} I_{s d}}{\partial V_{s d} \partial y} y_{R}\left(\omega_{i}\right)
$$

using

$$
y_{R}\left(\omega_{i}\right)=y_{R}\left(\omega_{c}\right)+y_{R}^{\prime}\left(\omega_{c}\right) \omega_{\Delta} \cos \left(\omega_{L} t\right)
$$

we finally have

$$
y d V: \frac{A T}{2} \frac{\partial^{2} I_{s d}}{\partial V_{s d} \partial y} y_{R}^{\prime}\left(\omega_{c}\right) \omega_{\Delta}
$$

The important result is that for FM the demodulated $I_{s d}$ is simply proportional to $y_{R}^{\prime}$.

Experiment and Results : Single-clamped FE nanoradio

Equations 14-17 and 18-20 give the different contributions of the demodulated current as a function of the field emission and mechanical properties of the CNT, the CNT environment and the RF parameters. Since many parameters are needed to describe the system, large order of magnitude errors in the estimation of the demodulated current can easily occur and consequently an important effort must be made to measure and/or estimate them well. In-situ experiments of the nanoradio were carried out in a TEM on different CNTs to obtain experimental values and good approximations of all the terms.

We used arc-discharge MWNTs glued at the apexes of tungsten tips. These CNT-tips were then mounted in a home-made TEM sample holder to simultaneously observe the motion of the CNTs and the demodulation process. The CNT-tip was connected to a heating loop that 

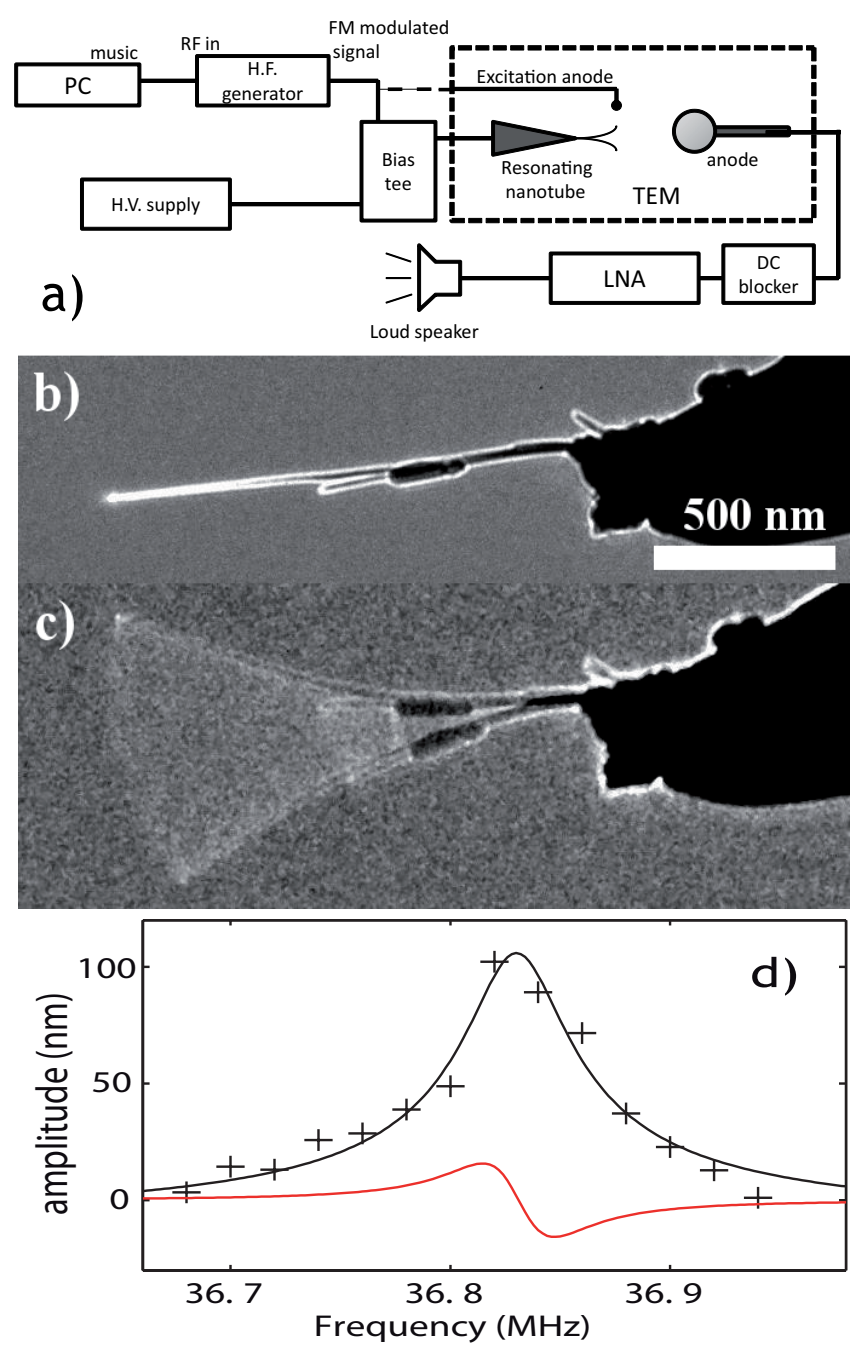

FIG. 3: (Color online) a) Schematic showing the configuration used for demodulation. b) Observation of one mutiwalled CNT used for these experiments. The CNT has a length of $1.3 \mu \mathrm{m}$ and a diameter of nearly $30 \mathrm{~nm}$. c) Excitation of the first resonance frequency. Large vibration amplitudes up to $400 \mathrm{~nm}$ can be obtained. d) Experimental (cross points) and fitted (black curve) amplitude frequency response of the CNT presented in figure 3 at zero applied voltage. This gave a resonance frequency of $36.83 \mathrm{MHz}$ and a quality factor 800 . From This curve allows the calculation of the the first derivative, $y^{\prime}\left(\omega_{c}\right)$, presented in of the real part of the response function (red curve - not to scale).

could be floated to high voltage $(2000 \mathrm{~V})$ and positioned near a gold ball that is both the extraction anode and the emission current collector. This anode was mounted on a micrometric movement that could be used to change the nanotube-anode distance. RF signals were sent to either the tip, the gold ball or the excitation electrode depending on the experimental conditions (see $3(\mathrm{a})$ ). This TEM holder was made to be inserted into a TOPCON EM002b microscope with large gap pole pieces which limit the resolution of the images to about $0.4 \mathrm{~nm}$ at $120 \mathrm{kV}$.

Preliminary observations were then realized to charac- terize the CNT dimensions (fig. 3b)). The FE performances of the CNT were first determined. The emission voltage is extremely sensitive to the nanotube-anode distance and nanotube dimensions. To partially normalize the experiments on different nanotubes the anode position was adjusted to obtain a current of $10 \mu \mathrm{A}$ for nearly $300 \mathrm{~V}$. Once this distance was established, the system was kept constant. The I(V) characteristics were shown to follow the FN equation and were fitted to find the parameters for our nanoradio. Using a work function, $\phi$, of $5 \mathrm{eV}$ and taking $\mathrm{B} \simeq-0.683 \phi^{3 / 2} v(y) \simeq 6.1110^{10} \mathrm{~V} \cdot \mathrm{m}^{-1}$ the experimental curves could be fitted to determine the amplification factor $\beta_{0}$ and the constant $\mathrm{C}$ in equation 12 (see Table I).

The resonance frequencies versus the applied voltage were determined next and the quality factor $\mathrm{Q}$ was measured at low excitation (see figure $3(\mathrm{~d})$ ). This gave the linear response of the cantilever that could be fit with a Lorentzian shape of the form stated in eq. 1. The CNT presented in figure 3 had a resonant frequency of $f_{0}=$ $\omega_{0} / 2 \pi=36.83 \mathrm{MHz}$ at zero applied voltage, a quality factor of around 800, that is rather large, and $T=3.95$ $10^{6} \mathrm{~m} . \mathrm{s}^{-2}$. This gives both the amplitude frequency response, $y\left(\omega_{c}\right)$ and its first derivative $y^{\prime}\left(\omega_{c}\right)$. Very high amplitudes, up to $400 \mathrm{~nm}$, could be obtained as seen in figure 3c) which was interesting for getting good electromechanical demodulation performance.

After the CNT was fully characterized the nanoradio experiments were carried out. A Rohde\&Schwarz SML 01 signal generator was used that permitted AM or FM modulation from external ports. We applied a DC voltage of 300 Volt giving an emission current in the $\mu \mathrm{A}$ range and found a frequency electrostatically tuned to $82 \mathrm{MHz}$. The RF modulated signal was then sent to the nanotube for demodulation and the carrier frequency was swept to obtain the best demodulation rate. The emission current was collected at the anode and sent, via a DC block to remove the DC current, to a low noise transimpedance amplifier (with a frequency cutoff at $100 \mathrm{kHz}$ ). The amplified signal was finally connected to a loud speaker for signal restitution (see figure 3 a)).

Demodulation of an AM signal was rather easy to obtain for the first tests using $h=0.9$ and $\mathrm{V}_{A C}=0.2 \mathrm{~V}$. Away from the resonance no signal was distinguishable although the $d V^{2}$ term should demodulate at any frequency. To distinguish this term from a large noise signal it was necessary to increase the signal amplitude to $\mathrm{V}_{A C}$ $\simeq 2 \mathrm{~V}$. This showed that this term is really negligible in the demodulation performance. Sweeping the carrier's frequency through the resonance gave a large and regular improvement of demodulation up to a maximum at the resonance frequency and a symmetric behavior for the other side of the resonance. As expected we found a single maximum in the demodulation signal. For $\mathrm{V}_{A C}=$ $0.2 \mathrm{~V}$ and $h=0.9$ we estimate a demodulated current of a few tens of $\mathrm{nA}$, since a $10^{8}$ amplification gain $(10 \mathrm{nA}$ gives $1 \mathrm{~V}$ ) was used.

In our FE experiments FM demodulation was clearly 
more difficult than AM in contrast to our recent work on a double-clamped nanoradio based on single wall nanotube transistors $^{9}$ (see below). For the same CNT it was necessary to increase the excitation voltages to obtain higher amplitudes of vibration in order to hear a distinguishable signal. The minimal values used to obtain an audible signal were $V_{A C}=0.3 \mathrm{~V}$ and $\omega_{\Delta}=2 \pi \times 10 \mathrm{kHz}$. With such a strong excitation signal, the nanotube was driven to a nonlinear Duffing regime with hysteresis and jumps. In this regime, we found only one maximum of demodulation. The demodulation current was estimated in the range of a few $\mathrm{nA}$.

The signal quality is limited by three main effects. Firstly the instability of the field emission is an important source of noise that is created by atomic diffusion and flip-flop movement on the emitter surface ${ }^{25}$. Some large changes in the current also appeared, in the $\mu \mathrm{A}$ range, due to formation or disappearance of emitting zones (nanoprotrusions) at the apex of the CNT. Normally such strong instabilities would tend to disappear during long emission experiments but the medium vacuum in the TEM $\left(\simeq 10^{-7}\right.$ torr $)$ compared to the ultra high vacuum generally used for FE experiments $\left(\simeq 10^{-10}\right.$ torr) does not promote this stabilization effect. For AM experiments, the higher demodulated currents hide the FE instabilities, but in FM experiments this noise was still significant. The second effect that reduces signal quality is the strong distortion of the signal. This is inherently related to the demodulation process where the mechanical AM variation is multiplied by itself thus leading to harmonic distortion from $2 \omega_{L}$ components. For a complex signal where several frequencies are present we also have intermodulation distortion. This will form additional signals at frequencies that are not, in general, at harmonic frequencies (integer multiples) of either, but instead often at sum and difference frequencies of the original frequencies. The third effect did a slow decreasing frequency shift of the resonance that altered the experiments, probably due to mass deposition generated by the TEM electron beam.

All the studied nanotubes did not show the same efficiency for demodulation. In particular some CNTs were difficult to excite and higher excitation voltages were necessary to obtain enough amplitude of vibration for distinguishable demodulation. The different reasons for this are discussed below as well as how the efficiency of excitation can be improved to obtain large amplitudes.

To simulate the performance of the FE nanoradio the only missing parameter is $\beta(y)$. i.e. $\beta_{1}$ and $\beta_{2}$ in eq. 10. Systematic numerical simulations were made to determine these parameters taking into account the deformation of the nanotube. $\beta(y)$ can be obtained from the normalized simulations multiplied by the experimental $\beta_{0}$. Simulations have been realized using a free and powerful finite element solver, Cast $3 \mathrm{M}^{26,27}$, adapted to treat electrostatic problems. The model consists of a CNT of length and radius comparable to those of the experiments positioned at the apex of a tip. The simulated nanotube

\begin{tabular}{|c|c|}
\hline Parameters & values \\
\hline $\mathrm{V}_{A}$ & $300 \mathrm{~V}$ \\
\hline $\mathrm{I}_{0}$ & $8 \mu \mathrm{A}$ \\
\hline $\mathrm{B}$ & $6.1110^{10} \mathrm{~V} \cdot \mathrm{m}^{-1}$ \\
\hline $\mathrm{C}$ & $1.21210^{-22} \mathrm{~A} . \mathrm{V}^{-1} \cdot \mathrm{m}^{-1}$ \\
\hline$\beta_{0}$ & $2.90510^{7} \mathrm{~m}^{-1}$ \\
\hline$\beta_{1}$ & $-9.353910^{11} \mathrm{~m}^{-2}$ \\
\hline$\beta_{2}$ & $-2.576510^{18} \mathrm{~m}^{-3}$ \\
\hline $\mathrm{Q}$ & 800 \\
\hline$\omega_{0}$ & $2 \pi \times 82.0 \mathrm{MHz}$ \\
\hline$\omega_{\Delta}$ & $2 \pi \pi^{*} 10 \mathrm{kHz}$ \\
\hline $\mathrm{A}$ & $0.3 \mathrm{~V}$ \\
\hline $\mathrm{h}$ & 0.9 \\
\hline$T$ & $4.9310^{7} \mathrm{~m} . \mathrm{s}^{-2}$ \\
\hline
\end{tabular}

TABLE I: Parameters used in the simulations on the demodulation performances of the FE configuration.

is mounted with a tilt angle of $10^{\circ}$ with respect to the support tip axis. This agrees with the experiments and gives a non-zero value for $\beta_{1}$. From this equilibrium position the nanotube is deformed according to the analytical solution for the first mode of vibration. For each deformation Cast3m solves the electrostatic problem. $\beta(y)$ for the center of the cap is followed as a function of apex position.

$\beta(y)$ varies quadratically by $\sim 1 \%$ during oscillations for an end angle $\theta \pm 20^{\circ}$. It is asymmetric because of the starting tilt and is maximum for a deflexion of nearly $200 \mathrm{~nm}$ when the CNT lines up with the support tip axis. For a CNT on a planar surface in the same configuration $\beta(y)$ varies more strongly $(\sim 5 \mathrm{x})$. This is due to the stronger screening effect of the underneath flat substrate compared to the support tip. This last shows that the specific geometry close to the cantilever is important for optimizing the demodulation.

The values of $\beta_{1}$ and $\beta_{2}$ were deduced for these geometries by the $\beta(y)$ curves and using the experimental values of $\beta_{0}$ (see Table 1 ). Finally, to have a amplitude of vibration of $300 \mathrm{~nm}$ comparable to Fig. 3c) we estimate $T=4.9310^{7} \mathrm{~m} . \mathrm{s}^{-2}$ in eq. 1 (assuming the same Q $=800)$. $T$ could have been estimated by $3 \mathrm{D}$ simulations. It is due to the imbalance of surface forces between opposing sides of the CNT. A simple formula has not yet been derived but $T$ is an increasing function of $\beta_{0}$ and the geometrical asymmetry, eg. tilt angle, which to first order also determines $\beta_{1}$. Thus for the sake of argument we write $T \alpha \beta_{0} \beta_{1}$.

All the necessary parameters for the next part of the simulations are summarized in table I. With these parameters eqs. 14 to 17 and eqs. 18 to 20 can be used to calculate the different demodulation components assuming linear mechanical response.

Figure 4 presents the total demodulated current and the different contributions from an AM RF signal versus the carrier frequency. As expected we obtain a maximum signal at the resonance frequency reaching $120 \mathrm{nA}$ which is in the range of our experiments. The main demodulat- 


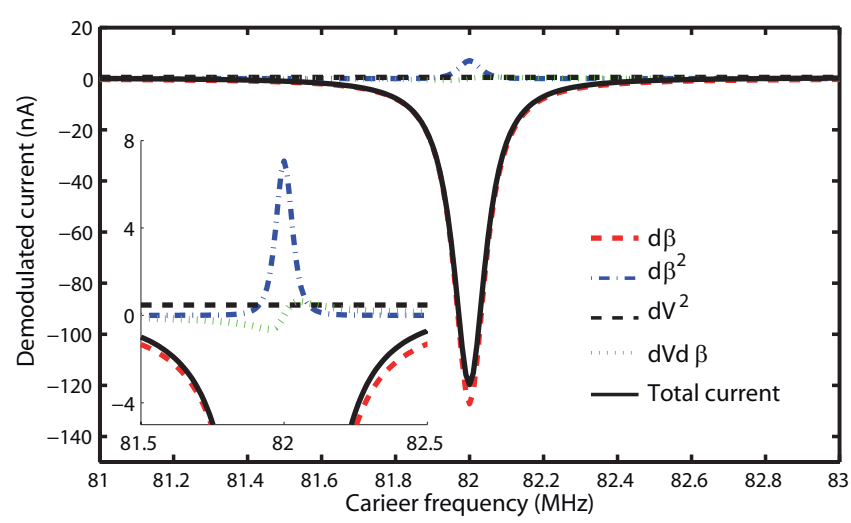

FIG. 4: (Color online) Calculated demodulation currents for an AM signal versus the carrier frequency. Clear maxima at the resonance frequency of the CNT are observed. Most of the demodulation comes from the $d \beta$ term which makes up almost all of the signal. The $d \beta^{2}$ term is the second largest which is of opposite sign, thus reducing slightly the overall demodulation current. The inset is a magnification to better show the smaller and almost negligible contributions.

ing term is clearly the $d \beta$, and thus, the $\beta_{2} y^{2}$ dependance of the field amplification factor. All the other terms are negligible except the $d \beta^{2}$ term but as it is of opposite sign compared to the $d \beta$ term, it tends to reduce the overall demodulation performances.

FM demodulation performances are presented in figure 5 for the same conditions. Two maxima are observed corresponding to the maximum slope of the response function. The maximum demodulated current is nearly 7 $\mathrm{nA}$, more than 10 times lower than AM demodulation. Once again, the $d \beta$ term is the main demodulating term and the $d \beta^{2}$ term decreases somewhat the demodulation. Two anti-symmetric peaks were obtained when in linear response but for the high amplitude non-linear response, only the first peak is present.

Further experiments with a lock-in amplifier to measure the experimental demodulated current versus the carrier frequency were made to compare with these calculations. Unfortunately that run of experiments led only to the destruction of samples or very noisy FE nanotubes so that only low quality data were obtained. Thus the above analysis still needs an experimental confirmation (demodulated current versus frequency and excitation voltage, etc.) but it already gives clear indications that signal optimization is obtained by maximizing the $d \beta$ term.

\section{Experiment and Results: Double-clamped SWNT transistor nanoradio}

The schematic for measuring the resonances of the SWNT in configuration 2 is shown in Fig. 6(a). A low frequency signal was sent from a lock-in amplifier to either the AM or FM inputs of a radio frequency (RF) generator. We typically used $\omega_{c}=616.3 \mathrm{~Hz}$ though audio signal frequencies were also tried. FM and AM signals

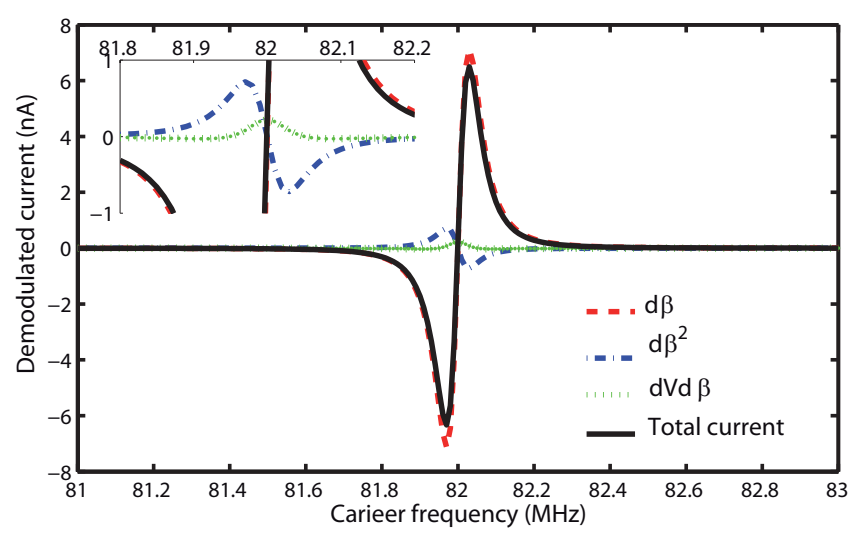

FIG. 5: (Color online) Demodulated current for a FM signal versus the carrier frequency. We observe two clear maximum at the resonance frequency of the CNT. The main demodulating term is the $d \beta$. The second term is the $d \beta^{2}$ but since it is of opposite sign it reduces the overall demodulation current. The inset presents a magnification to observe the other contributions that are presently negligible.

were applied to the source electrode of the nanotubes though FM turns out to be a much better technique. Finally the low frequency signal from the drain electrode was measured by the current input of the lock-in. The measurements were performed at room temperature in a home-made ultra high vacuum probe station. A high pass filter was used to filter low frequency leakage from the RF generator.

The device fabrication was as follows. CNTs were grown by chemical vapor deposition on a degenerated silicon substrate used as a back gate with a $300 \mathrm{~nm} \mathrm{SiO}_{2}$ layer. Contact pads for source and drain were fabricated by optical lithography, evaporation of $20 \mathrm{~nm}$ of $\mathrm{Cr}$ and $250 \mathrm{~nm}$ of gold and lift off. The spacing between electrodes is $2 \mu \mathrm{m}$ and the diameters of the tubes (measured by atomic force microscopy) were between 1 and 3 $\mathrm{nm}$. Finally, the tubes are suspended by a wet etching in buffered hydrofluoric acid followed by a critical point drying in $\mathrm{CO}_{2}$. Figure Fig. 6(b) shows a scanning electron microscope (SEM) image of a suspended nanotube at the end of the process.

Measurements were carried out on 7 different devices. We swept $\omega_{c}$ over large frequency spans and used the lock-in to record the magnitude $R$ and phase $\theta$ or " $X$ and $Y$ " measurements, where $X=R \cos (\theta)$ is the inphase current, and $X=R \cos (\theta)$ is the out-of-phase current. The signals were recorded for different fixed DC gate voltages.

Peaks in the current were found at various frequencies. These peaks were proven to be due to the vibrations of the nanotube by varying the DC gate voltage to tune the resonance frequencies ${ }^{4}$. Some measurements were performed with the same mixing technique as ref. ${ }^{4}$ to check that the resonance peaks of our sample are at the same frequency with both methods. After coarse identification of the resonances, finer frequency scans were made 

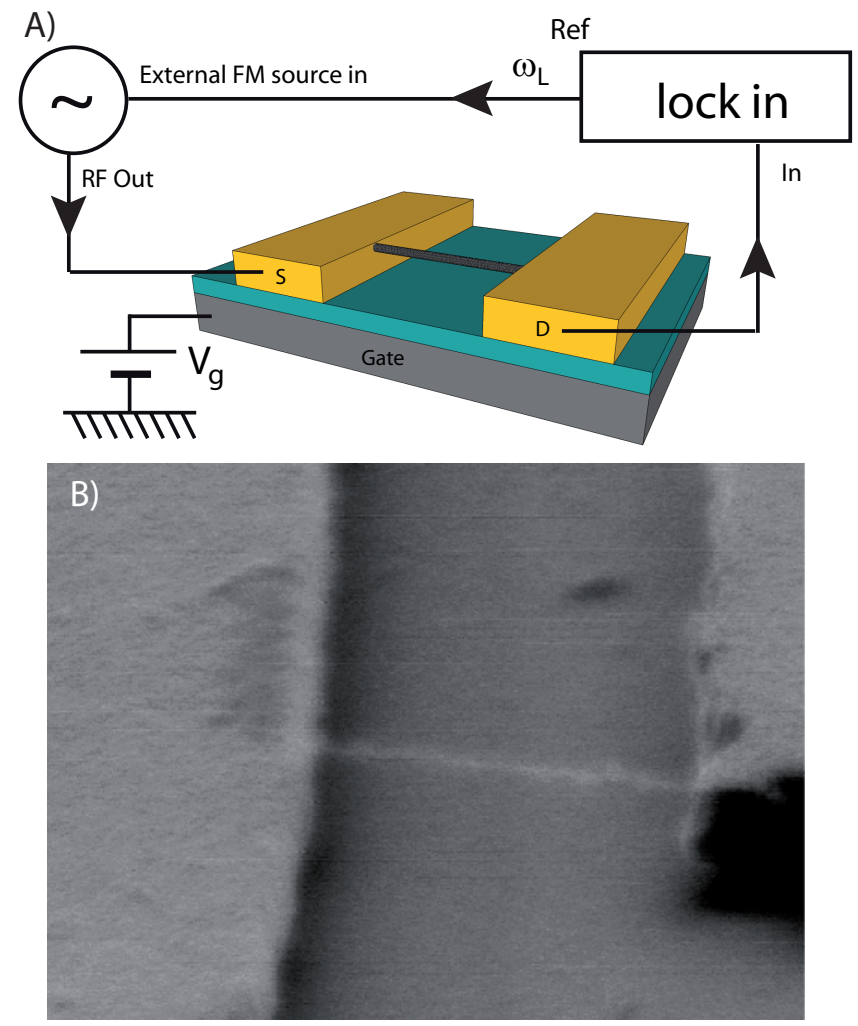

FIG. 6: a) Schematic showing the configuration used for demodulation. b) SEM image of a suspended SWNT transistor

to determine their exact shape. We performed AM demodulation at the resonance (see Figures 7 a) and b)) by applying at the drain an AM signal. The electromechanical signal is superimposed on a $25 \mathrm{pA}$ background current and the signal is rather noisy. For FM demodulation $\mathrm{X}$ (and R) had a rather unusual form with one central peak and two satellites. With an appropriate choice of the phase of the lock-in, we could cancel the $\mathrm{Y}$ component to get only the resonant current in the $\mathrm{X}$ component (see Figures $7 \mathrm{c}$ ) and $\mathrm{d}$ )). $\mathrm{X}$ is simply the derivative of the real part of the response function of the resonator as predicted above. The data is fit extremely well with equations 3 and 26. The cancelation of $\mathrm{Y}$ is a specificity of FM detection. Thus the mathematics developed above is directly exploitable in radio applications. The low noise in X show that FM demodulation is a clear improvement in terms of mechanical detection which follows the known principle that FM systems are better in rejecting noise than AM systems ${ }^{28}$. Indeed, noise is generally related to amplitude variation and is nearly independent of frequency as long as the signal is not at too low a frequency.

An important point about the cancelation of the $\mathrm{Y}$ component is that this detection technique is not only sensitive to the frequency $\omega_{L}$, but also to its phase. This is of some interest for digital data transfer since some common modulation schemes such as QPSK (quadrature phase shift keying) uses the phase of the signal to enhance data transmission by sending more than 1 bit at the same
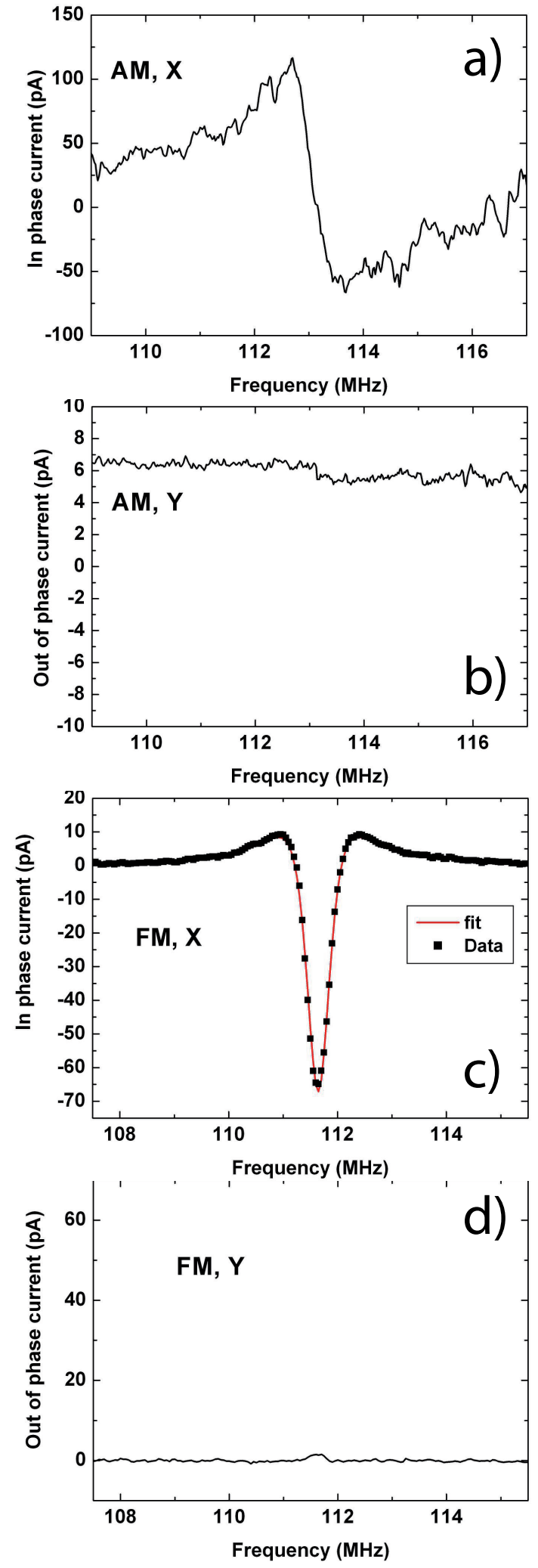

FIG. 7: a) and b): in-phase and out-of-phase lock-in current versus carrier frequency in $\mathrm{AM}$ mode for $\mathrm{m}=0.9$, $\omega_{L} / 2 \pi=616.3 \mathrm{~Hz}, V_{g}=-9.5$ Volt, $A=20 \mathrm{mV}$ and a time constant of $300 \mathrm{~ms}$. c) and d): in-phase and out-ofphase lock-in current versus carrier frequency in FM mode for $\omega_{\Delta} / 2 \pi=75 \mathrm{kHz}, \omega_{L} / 2 \pi=616.3 \mathrm{~Hz}, V_{g}=5 \mathrm{Volt}, A=10$ $\mathrm{mV}$ and a time constant of $100 \mathrm{~ms}$. The solid line in c) is a fit of the experimental data with Equation $26(Q=160$, $\left.\omega_{0} / 2 \pi=73.6 \mathrm{MHz}\right)$. 
time. The principle is to code, for example, 2 bits by changing the phase $\varphi$ by steps of $\pi / 2$ steps. This gives 4 different states $(\varphi=0$ coding 00 in bits, $\varphi=\pi / 2$ for 01 , $\varphi=\pi$ for 10 and $\varphi=\pi / 3 / 2$ for 11$)$.

Experimentally, we used an independent low frequency generator to send the $\cos \left(\omega_{L} t+\varphi\right)$ signal that FM modulates the carrier frequency of the high frequency generator. We recorded the demodulated signal in the lock-in using its internal source. The carrier frequency is fixed at the resonance of the nanotube. For $\varphi=0$, we set the phase of the lock-in, in order to have a large negative signal in $\mathrm{X}$ and zero in $\mathrm{Y}$, then we keep constant the phase of the lock-in and only change the phase of the external low frequency generator. So for $\varphi=\pi / 2$ we got zero in $\mathrm{X}$ and a large negative signal in $\mathrm{Y}$ i.e. the resonance is detected by the out of phase component. For $\varphi=\pi$ (respectively $\varphi=\pi / 3 / 2$ ) we got a sign reversal in $\mathrm{X}$ (respectively $\mathrm{Y}$ ) compared to $\varphi=0$ (respectively $\varphi=\pi / 2$ ). Figure 8 a) clearly demonstrates that such digital demodulation is possible for a nanotube nanoradio.

The maximum transfer rate about 60 bps (bit per second) is limited here by the $30 \mathrm{~ms}$ time transfer of our GPIB connection between the lock-in and the computer. To push the data transfer rate even further we i) used a sample with a resonant electromechanical current above $1 \mathrm{nA}$ to lower the time constant of the lock-in and still have a reasonable signal to noise ration and ii) did the measurement of the current with the analog $100 \mathrm{kHz}$ bandwidth output of the lock-in sent to a high speed oscilloscope. Figure $8 \mathrm{~b}$ ) shows a rise time of $200 \mu \mathrm{s}$ with a signal to noise ration of 8 when passing from 00 to 01 . Thus we have achieved a transfer rate of $10 \mathrm{kbps}$ thanks to this 2 bit at a time transfer method. This means that our device reached the requirement for the widely used GSM (global system for mobile communication) cell phone standard where the voice is digitally transported at a $9.6 \mathrm{kbps}$ rate. Higher rates would require higher demodulation current which could be obtained by reducing damping from clamping, nanotube surfaces or temperature effects.

\section{Discussion}

The two configurations have certain similarities and differences that are worth discussing. Firstly, just because the FE and transistor nanoradios were carried out in vastly different environments doesn't mean they involve very different physics. Though the first geometry is more easily associated to electron sources for vacuum tube science and the second to research in micro-nanoelectronics, the similarities can be seen immediately by inspecting Fig. 1. In both cases the measured signal is a current that transits the nanotube cantilever, though one is controlled by the field-dependent tunnel barrier and the other by the field-dependent volume conductivity. The current in both cases depends on the transverse position of the cantilever $y(t)$, the amplitude of the RF signal and a static voltage that controls the resonant frequency.
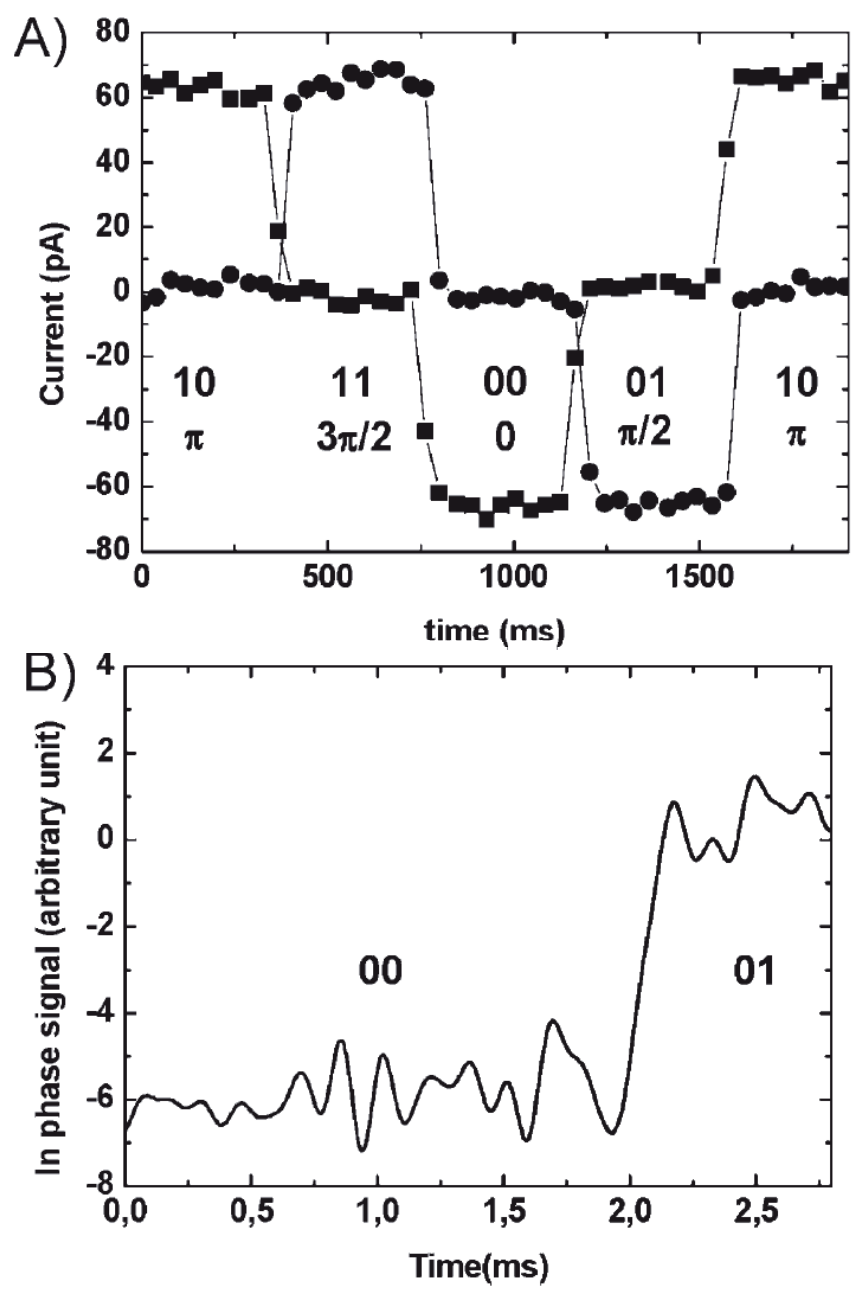

FIG. 8: a) Schematic Digital demodulation ( $\mathbf{\square}$ in phase and - out of phase component) for $\omega_{\Delta} / 2 \pi=200 \mathrm{kHz}, \omega_{L} / 2 \pi=$ $616.3 \mathrm{~Hz}, \omega_{c} / 2 \pi=112.8 \mathrm{MHz}, V_{g}=-9.5 \mathrm{~V}, \mathrm{~A}=20 \mathrm{mV}$ and a time constant of $10 \mathrm{~ms}$. B) High speed digital demodulation for $\omega_{\Delta} / 2 \pi=750 \mathrm{kHz}, \omega_{L} / 2 \pi=7243 \mathrm{~Hz}, \omega_{c} / 2 \pi=$ $156.98 \mathrm{MHz}, V_{g}=9 \mathrm{~V}, A=20 \mathrm{mV}$ and a time constant of $30 \mu s$.

We can write in general $I(y(t), R F$ signal, $\omega(V$ control $)$. Though difficult in practise, in principle the $\mathrm{FE}$ version could be made by simply cutting the CNT of the transistor version. Finally both experiments are carried out in vacuum to have a reasonable $\mathrm{Q}$ factor and a stable resonance frequency.

The distinguishing aspects of the two configurations are summarized in Table II. One of the principle differences is that the present FE version is a diode and the transistor is a triode. The FE voltage has thus two roles. It varies both $I_{F E}$ and the resonance frequency simultaneously. This is a serious constraint during operation. In the transistor nanoradio $V_{g}$ tunes $\omega_{0}$ quasi-independently of $I_{s d}$ at $V_{s d}=0$. Several other differences are i) FE usually requires high voltage (typically 100's of Volts). The high voltage would be reduced in a cut CNT design but generally would not be less than $\sim 50 \mathrm{~V}$. ii) FE requires 


\begin{tabular}{|l|l|l|}
\hline Parameter & FE configuration & $\begin{array}{l}\text { transistor } \\
\text { configuration }\end{array}$ \\
\hline operating voltage & $\mathrm{V}_{A}>100 \mathrm{~V}$ & $\mathrm{~V}_{A}=0$ \\
\hline station selection & $\mathrm{V}_{A}>100 \mathrm{~V}$ & $\mathrm{~V}_{g}=$ few Volts \\
\hline RF signal & few 100 's $\mathrm{mV}$ & few 10 's $\mathrm{mV}$ \\
\hline vacuum & $\mathrm{UHV}$ & primary vacuum \\
\hline
\end{tabular}

TABLE II: Parameters used in the simulations on the demodulation performances

an even better vacuum because adsorbates cause large instabilities in the FE current. A vacuum of $10^{-3}$ Torr is sufficient to eliminate the air damping of the cantilever resonance but generally $10^{-8}$ Torr or better is needed for stable FE. An unstable FE current creates noise that passes through the down-mixing to the sound signal. In general FE is thought to be less compatible with microelectronics technology. The SWNT transistor has largely been developed in the context of new transistors for the microelectronics industry. However integrated CNT FE devices do exist ${ }^{21}$ though nanomechanical experiments have not been performed on them.

Despite these remarks the eventual deployment of the FE nanoradio in applications should not be too quickly discounted. In many ways it resembles the original vacuum tube radio of Armstrong and tube devices are still important in RF telecommunications. As well it uses the more robust MWNTs and vacuum packaging is widely practised.

Now let us consider the nanotube as an antenna that transforms an electromagnetic (em) wave into an electrical circuit signal or vice versa. We consider only FE configuration for which it was pointed out that the CNT gains energy from the electromagnetic wave and transforms it into a current wave at the mechanical resonance frequency. That is, the electric charge $q$ sitting on the CNT due to the static FE voltage is excited by the forces generated by the electromagnetic wave, following $f=q E$. In principle this is a new form of antenna. Unfortunately our estimation of the forces shows that the nanotube has a very weak response to the original em wave. Consider an em wave with a field $\mathrm{E}=0.2$ $\mathrm{V} / \mathrm{m}$ that corresponds to the electric field at one kilometer from an isotropic antenna of power $1 \mathrm{~kW}$. The charge on the CNT is obtained from the capacitance, C, of the CNT. Pushing the simulations one gets optimistically $\mathrm{C}$ $=10^{-16} \mathrm{~F}$. For $300 \mathrm{~V}$ that leads to a total charge of $\mathrm{q}$ $=310^{-14} \mathrm{C}$ and hence to a maximal force of $\mathrm{f}=610^{-15}$ $\mathrm{N}$. Assume the most favorable case that all this force is transverse to the CNT axis and located at the apex of the CNT. Using an equivalent spring constant for the first mode of $\mathrm{k}=10^{-2} \mathrm{~N} / \mathrm{m}$ this leads to a static deflexion of $\mathrm{x}_{s}=610^{-13} \mathrm{~m}$. At the resonance one gets only $\mathrm{x}_{r}=$ $\mathrm{Q} \mathrm{x}_{s}=610^{-10}=0.6 \mathrm{~nm}$ ! Thus, even highly overestimated, the direct excitation of the CNT by a reasonable magnitude em wave is too small to produce a perceptible variation of the field emission current.

\section{Conclusion}

This article attempts to present a fairly complete set of equations and experiments for AM and FM demodulation by CNT FE and transistor nanoradios. The demodulation performances versus the carrier frequency for different terms in the Taylor expansion were calculated. Experimental measurements and realistic estimations of the necessary system parameters have been made.

It was shown that for the FE nanoradio most of the demodulation effect comes from $\beta(y)$, the dependence of the field amplification factor on the position of the CNT cap. In particular the second order derivative, $\beta_{2}$ is shown to be dominant in contrast to previous work ${ }^{6}$. Experimentally and theoretically AM leads to larger modulation currents than FM $(\sim \mathrm{x} 10)$ for the explored range of parameters, though it may be possible to inverse this trend for a different choice of parameters.

Estimations of the excitation efficiency of a charged CNT for capturing RF signals have been made and show that this new type of antenna works very poorly in the open FE geometry, that is for a CNT on a tip. In other words this new type of mechanical reception does not circumvent the basic difficulty of the mismatch between the $\mathrm{RF}$ wavelength and CNT length. For the capacitive excitation the coupling between the excitation electrode and the CNT must be as asymmetrical as possible. Such an excitation electrode can be a coplanar wideband waveguide connected to an external antenna and terminated as close as possible to the emitting nanotubes. A better optimization taking into account the electrostatic, electromagnetic, mechanical and FE is an engineering problem that must be tackled before the real capabilities and characteristics of this nanoradio are known.

For the SWNT transistor nanoradio we showed that $\mathrm{AM}, \mathrm{FM}$ and digital demodulation is possible. We theoretically demonstrated that the shape of the resonance peak in FM is proportional to the derivative of the real part of the response function of the resonator. With this technique, it is possible to reduce the noise and unwanted background signals. The noise reduction of FM demodulation might be useful for NEMS applications such as ultimate mass sensing. It is becoming the method of choice having for example been recently used for graphene oscillators ${ }^{29}$. Our device achieved a transfer rate of $10 \mathrm{kbps}$ compatible with GSM requirement. This work describes experiments on carbon nanotubes. However both semiconducting nanowires and graphene are being explored as resonators. They may turn out to have advantages over the SWNTs in nanoradio applications, for example they are more amenable to parallel top-down fabrication ${ }^{29}$.

\section{Acknowledgments}

This work was supported by French National Research Agency (ANR) through its Nanoscience and Nanotechnology Program (NEXTNEMS, ANR-07-NANO-008-01) and Jeunes Chercheuses et Jeunes Chercheurs Program 
(AUTONOME, ANR-07-JCJC- 0145-01). The authors acknowledge the "Plateforme Nanofils et Nanotubes Ly- onnaise" of the university Lyon1.
* Electronic address: pascal.vincent@univ-lyon1.fr

1 J.M. Kahn, R.H. Katz, K.S. Pister, J. Commun.Networks 2, 188 (2000).

2 P. Poncharal, Z. L. Wang, D. Ugarte and W. A. de Heer, Science 283 1513-1516 (1999).

3 S.T. Purcell, P. Vincent, C. Journet and V. T. Binh, Phys. Rev. Lett. 89, 276103 (2002).

${ }^{4}$ V. Sazonova, Y. Yaish, H. Üstünel, D. Roundy, T. A. Arias, and P. L. McEuen, Nature 431, 284 (2004).

5 S. Sapmaz, Ya. M. Blanter, L. Gurevich, and H. S. J. van der Zant, Phys. Rev. B 67, 235414 (2003).

${ }^{6}$ K. Jensen, H. Garcia and A. Zettl, Nano Letters 7, 3508 (2007).

7 S. Perisanu, A. Ayari, S.T. Purcell, P. Vincent, Int. Journ. of Nanotechnology 7, 702 (2010).

${ }^{8}$ C. Rutherglen, P. Burke, Nano Letters 7, 3296-3299 (2007).

9 V. Gouttenoire, T. Barois, S. Perisanu, J.L. Leclercq, S.T. Purcell, P. Vincent, A. Ayari, Small, 6, 1060 (2010).

10 P. Vincent, A. Ayari, P. Poncharal, T. Barois, S. Perisanu, and S.T. Purcell, Phys. Rev. B (submitted).

11 C.K. Yanga, A.J. le Fèbre, G. Pandraud, E. van der Drift, P.J. French, J. Vac. Sci. Technol. B 26, 927 (2008).

12 K. Jensen, K. Kim and A. Zettl, Nature Nanotechnology 3, 533 (2008).

13 A. Ayari, P. Vincent, S. Perisanu, M. Choueib, V. Gouttenoire, M. Bechelany, D. Cornu and S.T. Purcell, Nano Letters 7, 2252 (2007).

${ }^{14}$ H.S. Kim, H. Qin, M.S. Westphall, L.M. Smith, R.H. Blick, Nanotechnology 18, 065201 (2007).

15 Z. Wang, J. Wei, P. Morse, J.G. Dash, O.E. Vilches, and D.H. Cobden, Science 328 552-555 (2010).

16 B. Lassagne, Y. Tarakanov, J. Kinaret, D. Garcia-Sanchez and A. Bachtold, Science 325 1107-1110, (2010).

17 G.A. Steele, A.K. Httel, B. Witkamp, M. Poot, H. B. Meerwaldt, L.P. Kouwenhoven and H.S.J. van der Zant Science
325 1103-1107, (2010).

18 M. F. Yu, G. J. Wagner, R. S. Ruoff, and M. J. Dyer, Phys. Rev. B 66, 073406 (2002).

19 S. Perisanu, P. Vincent, A. Ayari, M. Choueib, S. T. Purcell, M. Bechelany, and D. Cornu, Appl. Phys. Lett. 90, 043113 (2007).

20 S. Perisanu, V. Gouttenoire, P. Vincent, A. Ayari, M. Choueib, M. Bechelany, D. Cornu, and S. T. Purcell, Phys. Rev. B 77, 165434 (2008).

${ }^{21}$ L. Gangloff, E. Minoux, K.B.K. Teo, P. Vincent, V. Semet, V.T. Binh, M.H. Yang, I.Y.Y. Bu, R.G. Lacerda, G. Pirio, J.P. Schnell, D. Pribat, D.G. Hasko, G.A.J. Amaratunga, W.I. Milne, and P. Legagneux, Nano Letters 4, 1575-1579 (2004).

22 It is easy to show that $\left.\frac{\partial I}{\partial V}\right|_{V_{A}}=I_{0}\left(\frac{2}{V_{A}}+\frac{B}{\beta_{0} V_{A}^{2}}\right),\left.\frac{\partial I}{\partial \beta}\right|_{\beta_{0}}=$ $I_{0}\left(\frac{2}{\beta_{0}}+\frac{B}{\beta_{0}^{2} V_{A}}\right),\left.\frac{\partial^{2} I}{\partial V^{2}}\right|_{V_{A}}=I_{0}\left(\frac{2}{V_{A}^{2}}+\frac{2 B}{\beta_{0} V_{A}^{3}}+\frac{B^{2}}{\beta_{0}^{2} V_{A}^{4}}\right),\left.\frac{\partial^{2} I}{\partial \beta^{2}}\right|_{\beta_{0}}=$ $I_{0}\left(\frac{2}{\beta_{0}^{2}}+\frac{2 B}{\beta_{0}^{3} V_{A}}+\frac{B^{2}}{\beta_{0}^{4} V_{A}^{2}}\right)$ and $\left.\frac{\partial^{2} I}{\partial \beta \partial V}\right|_{V_{A}, \beta_{0}}=I_{0}\left(\frac{4}{\beta_{0} V_{A}}+\frac{3 B}{\beta_{0}^{2} V_{A}^{2}}+\right.$ $\left.\frac{B^{2}}{\beta_{0}^{3} V_{A}^{3}}\right)$. where $I_{0}=I_{F E}\left(V_{A}, \beta_{0}\right)$

23 E.D. Minot, Y. Yaish, V. Sazonova, J. Park, M. Brink, P.L. McEuen, Phys. Rev. Lett. 90, 156401 (2003).

24 J. Cao, Q. Wang, H. Dai, Phys. Rev. Lett. 90, 157601 (2003).

25 R. Gomer, Surface Science, 38, 373 (1973).

${ }^{26}$ website : http://www-cast3m.cea.fr/cast3m

27 P. Moin, Fundamentals of engineering. numerical analysis, Cambridge: Cambridge University Press (2001).

${ }^{28}$ D. Rutledge, The electronics of radio, Cambridge University Press, 1999, p. 9.

29 A.M. van der Zande, R.A. Barton, J.S. Alden, C.S. RuizVargas, W.S. Whitney, P.H.Q. Pham, J.Park, J.M. Parpia, H.G. Craighead, and P.L. McEuen, Nano Letters 10, 48694873 (2010). 